\title{
A Markovian single server working vacation queue with server state dependent arrival rate, balking and with breakdown
}

\author{
R. Kalyanaraman ${ }^{1 *}$ and A. Sundaramoorthy ${ }^{2}$
}

\begin{abstract}
A single server Markovian queueing system with the system alternates between regular busy state, repair state and working vacation state has been considered. When the system is busy, it functions as a single server Markovian queue. When it is on vacation, again it functions as a single server Markovian queue but with different arrival and service rates. The vacation policy is multiple vacation policy and the vacation period follows negative exponential. In addition, the customer may balk on arrival and during busy state the server may breakdown, the breakdown period follows negative exponential. Immediately, the repair takes place, repair period also follows negative exponential. The steady state probability vector of number of customers in the queue and the stability condition are obtained using Matrix-Geometric method. Some illustrative examples are also provided.
\end{abstract}

\section{Keywords}

Working vacation, State dependent arrival rate, Matrix-Geometric method.

\section{AMS Subject Classification}

90B22, 60K25 and 60K30.

1,2 Department of Mathematics, Annamalai University, Annamalainagar-608002, India.

${ }^{*}$ Corresponding author: ${ }^{1}$ r.kalyan24@rediff.com; ${ }^{2}$ a.sundarmaths92@gmail.com

Article History: Received 24 March 2019; Accepted 09 May 2019

\section{Contents}

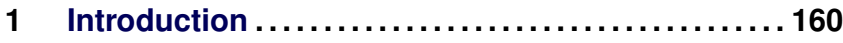

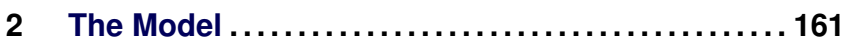

2.1 The quasi-birth-and-death(QBD) process ... 162

3 The Steady State Solution .................... 162

4 Performance Measures ..................... 163

5 Particular Model..........................164

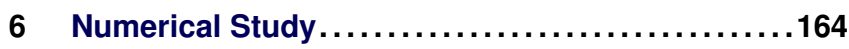

7 Conclusion .................................. 165

References .165

\section{Introduction}

In many real life queueing situations, it can be seen that the server works during his rest period, if the necessity occur. This leads to a new class of queueing systems called queueing systems with server working vacation. This type of queues can be used to modeled the situations in telecommunication systems and manufacturing systems. Queueing systems with server vacations have been investigated by many researchers, the readers may refer the survey paper by [6] and the monograph of [26].

In the working vacation queues, the server works with variable service rate, in particular reduced service rate, rather than completely stops service during vacation period. [22] have first analyzed an $M / M / 1$ queue with multiple working vacation, in which the vacation times are exponentially distributed. [32] extend the work for $M / G / 1$ queue. [14] analyzed the queue length distribution of the $M / G / 1$ queue with working vacations. [18], examined stochastic decomposition structure of the queue length and waiting time in an $M / M / 1$ working vacation queue. [33] extended the $M / M / 1$ working vacation queue to an $M^{[x]} / M / 1$ working vacation queue. [16] used the matrix analytic method to analyze an $M / G / 1$ queue with exponential working vacation under a specific assumption. [17] consider a multi server queue with single working vacation. [11] investigated a single working vacation model with server break down. [13] have given a short survey on vacation models in recent years.

[34] have considered a single-server exponential queueing 
model with arrival state depending on operational state or breakdown state of the server. [8] analyzed the same model with the assumption that any arrival finding the server busy is lost, and they obtained the steady-state proportion of customer's lost. [25] has deals with a single server queueing model with arrival rate dependent on server state. [23] has analyzed a single server Poisson queue with arrival rate dependent on the state of the server. [12] analyzed a general bulk service queue with arrival rate dependent on server breakdowns. [28] discussed the queueing system with variable arrival rate. The author studied the model by using the principle of quasi-birth and death process(QBD) and matrix-geometric method. Furthermore, the calculated some performance measures, such as the number of customers in the system in steady-state. Matrixgeometric method approach is a useful tool for solving the more complex queueing problems. Matrix-geometric method has been applied by many researchers to solve various queueing problems in different frameworks. [21] explained various matrix geometric solutions of stochastic models. Matrixgeometric approach is utilized to develop the computable explicit formula for the probability distributions of the queue length and other system characteristic.

It can be commonly seen that some customers may not enter the queue on finding the system down, the server absent from the system an presence of long queue. This type of impatient behavior of customer is called balking. For queues with balking are may refer [5], [10], [2], [3], [30] and [24].

In some queueing situations, it can be seen that systems can suddenly break down such us computer system, communication system, and many other. For example, consider a machine that always needs some maintenance otherwise it will be break down and a repair man must be called for repair. this type of queues are called queue with unreliable server. ([4], [9], [31], [27], [7] and [29]).

In this paper, we consider an $M / M / 1$ queue with multiple working vacation and balking and with breakdown. The arrival rate dependents on the server states. The model has been analyzed using matrix geometric method. The rest of this paper is organized as follows: In section 2, we give the model description, establish its quasi-birth-death process. In section 3 , we present the steady state solution using matrix geometric method . In section 4, we present some system performance measures. Section 5 gives some particular models. and In section 6 , we carried out a numerical study. Finally, a conclusion has been given.

\section{The Model}

We consider a single-server queueing system with the following characteristics:

(1) The system alternate between two states, up state and down state. In the up state it is either in regular state or in working vacation state. In the down state it is in the repair state.
(2) Arrival process follows Poisson with parameter $\lambda$ during regular state.

(3) When the system is in regular busy period it serve customers based on exponential distribution with rate $\mu$.

(4) The server takes vacation, if there are no customer in the queue at a service completion point.

(5) During vacation, the arrival follows Poisson with rate $\lambda_{1}\left(\lambda_{1}<\lambda\right)$.

(6) Vacation period follows negative exponential with rate $\theta$ and the vacation policy is multiple vacation policy.

(7) When the server is in vacation, if customer arrives, the server serve the customers using exponential distribution with rate $\mu_{1}\left(\mu_{1}<\mu\right)$. As this vacation period ends, the server instantaneously switches over to the normal service rate $\mu$, if there is at least one customer waiting for service. Upon completion of a service at a vacation period, the server will (i) Continue the current vacation if it is not finished and no customer is waiting for service; (ii) Continue the service with rate $\mu_{1}$ if the vacation has not expired and if there is at least one customer waiting for service.

(8) The server may break down during a regular busy period and the break downs are assumed to occur according to a Poisson process with rate $\alpha$.

(9) Once the system break downs, the customer whose service is interrupted goes to the head of the queue and the repair to server starts immediately.

(10) Duration of repaired period follows negative exponential with rate $\beta$.

(11) During repair period no service takes place but customers arrive according to Poisson process with rate $\lambda_{2}\left(\lambda_{2}<\lambda_{1}\right)$.

(12) If the customer finds the server in regular busy period, the customer joints system with probability $q$ and Balking with probability $(1-q)$.

(13) If the customer finds the server is an working vacation, the customer joints system with probability $q_{1}$ and Balking with probability $\left(1-q_{1}\right)$.

(14) If the customer finds the server is an repaired period, the customer joints system with probability $q_{2}$ and Balking with probability $\left(1-q_{2}\right)$.

(15) The first come first served (FCFS) service rule is followed to select the customer for service. 


\subsection{The quasi-birth-and-death(QBD) process}

The model defined in this article can be studied as a QBD process. The following notations are necessary for the analysis:

Let $L(t)$ be the number of customers in the queue at time $t$ and let

$$
J(t)=\left\{\begin{array}{l}
0, \text { if the server is on working vacation } \\
1, \text { if the server is busy } \\
2, \text { if the server is on repaired period }
\end{array}\right.
$$

be the server state at time $t$.

Let $X(t)=(L(t), J(t))$, then $\{(X(t)): t \geq 0\}$ is a Continuous time Markov chain (CTMC) with state space $S=$ $\{(i, j): i \geq 0 ; j=0,1,2\}$, where $i$ denotes the number of customer in the queue and $j$ denotes the server state.

Using the lexicographical sequence for the states, the rate matrix $Q$, is the infinitesimal generator of the Markov chain and is given by

$$
Q=\left[\begin{array}{ccccccccc}
B_{0} & B_{1} & & & & & \\
A_{2} & A_{1} & A_{0} & & & & \\
& A_{2} & A_{1} & A_{0} & & & \\
& & A_{2} & A_{1} & A_{0} & & \\
& & & \cdot & . & . & \\
& & & & \cdot & . & . \\
& & & & & . & . & .
\end{array}\right]
$$

where the sub-matrices $A_{0}, A_{1}$, and $A_{2}$ are of order $3 \times 3$ and are appearing as

$$
\begin{aligned}
& A_{0}=\left[\begin{array}{ccc}
q_{1} \lambda_{1} & 0 & 0 \\
0 & q \lambda & 0 \\
0 & 0 & q_{2} \lambda_{2}
\end{array}\right] \\
& A_{1}=\left[\begin{array}{ccc}
-\left(q_{1} \lambda_{1}+\mu_{1}+\theta\right) & \theta & 0 \\
0 & -(q \lambda+\mu+\alpha) & \alpha \\
0 & \beta & -\left(q_{2} \lambda_{2}+\beta\right)
\end{array}\right] \\
& A_{2}=\left[\begin{array}{ccc}
\mu_{1} & 0 & 0 \\
0 & \mu & 0 \\
0 & 0 & 0
\end{array}\right]
\end{aligned}
$$

and the boundary matrices are defined by

$$
\begin{aligned}
& B_{0}=\left[\begin{array}{ccc}
-\left(\lambda_{1}+\theta\right) & \theta & 0 \\
\mu & -(q \lambda+\mu+\alpha) & \alpha \\
0 & \beta & -\left(q_{2} \lambda_{2}+\beta\right)
\end{array}\right] \\
& B_{1}=\left[\begin{array}{ccc}
\lambda_{1} & 0 & 0 \\
0 & q \lambda & 0 \\
0 & 0 & q_{2} \lambda_{2}
\end{array}\right]
\end{aligned}
$$

\section{The Steady State Solution}

Let $P=\left(p_{0}, p_{1}, p_{2}, \ldots\right)$ be the stationary probability vector associated with $Q$, such that $P Q=0$ and $P e=1$, where $e$ is a column vector of $1^{\prime} s$ of appropriate dimension.

Let $p_{0}=\left(p_{00}, p_{01}, p_{02}\right), p_{1}=\left(p_{10}, p_{11}, p_{12}\right)$ and $p_{i}=\left(p_{i 0}, p_{i 1}, p_{i 2}\right)$ for $i \geq 2$.

If the steady state condition is satisfied, then the sub vectors $p_{i}$ are given by the following equations:

$$
\begin{array}{r}
p_{0} B_{0}+p_{1} A_{2}=0 \\
p_{0} B_{1}+p_{1} A_{1}+p_{2} A_{2}=0 \\
p_{i} A_{0}+p_{i+1} A_{1}+p_{i+2} A_{2}=0, i \geq 1 \\
p_{i}=p_{1} R^{i-1} ; i \geq 2
\end{array}
$$

where $R$ is the rate matrix, is the minimal non-negative solution of the matrix quadratic equation (see [21]).

$$
R^{2} A_{2}+R A_{1}+A_{0}=0
$$

Substituting the equation (3.4) in (3.2), we have

$$
p_{0} B_{1}+p_{1}\left(A_{1}+R A_{2}\right)=0
$$

and the normalizing condition is

$$
p_{0} e+p_{1}(I-R)^{-1} e=1
$$

Theorem 3.1. The queueing system described in the article is stable if and only if $\rho<1$, where $\rho=\frac{q \lambda \beta+q_{2} \lambda_{2} \alpha}{\mu \beta}$

Proof. Consider the infinitesimal generator

$$
A=\left[\begin{array}{ccc}
-\theta & \theta & 0 \\
0 & -\alpha & \alpha \\
0 & \beta & -\beta
\end{array}\right], \text { which is a square matrix of order }
$$

3 , the row vector $\pi=\left(\pi_{0}, \pi_{1}, \pi_{2}\right)$ satisfying the condition $\pi A=0$ and $\pi e=1$.

That is,

The system is stable if and only if $\frac{q \lambda \beta+q_{2} \lambda_{2} \alpha}{\mu \beta}<1$

Theorem 3.2. If $\rho<1$, the matrix equation (3.5) has the minimal non-negative solution

$$
R=-A_{0} A_{1}^{-1}-R^{2} A_{2} A_{1}^{-1}
$$

Proof. We define the matrix $A=A_{0}+A_{1}+A_{2}$. This matrix $A$ is a $3 \times 3$ matrix and it can be written as

$$
A=\left[\begin{array}{ccc}
-\theta & \theta & 0 \\
0 & -\alpha & \alpha \\
0 & \beta & -\beta
\end{array}\right] \text { is reducible. The analysis present }
$$
in [20] is not applicable. In [19], similar reducible matrix is treated for the case when the elements are probabilities.

Equation (3.5), can be written as,

$$
A_{0} A_{1}^{-1}+R A_{1} A_{1}^{-1}+R^{2} A_{2} A_{1}^{-1}=0 A_{1}^{-1}
$$

Since $A_{1}$ is non-singular, $A_{1}^{-1}$ exists

$$
R=-A_{0} A_{1}^{-1}-R^{2} A_{2} A_{1}^{-1}
$$

Using [20] and [19], the matrix $R$ is numerically computed by using the recurrence relation with $R(0)=0$ in equation (3.8). 
Theorem 3.3. If $\rho<1$, the stationary probability vectors $p_{0}=\left(p_{00}, p_{01}, p_{02}\right), p_{1}=\left(p_{10}, p_{11}, p_{12}\right)$ and $p_{i}=\left(p_{i 0}, p_{i 1}, p_{i 2}\right)$ for $i \geq 2$ are

$$
\begin{aligned}
& p_{00}=\left[1+\frac{S_{0} \lambda_{1}}{\left[\left(q_{1} \lambda_{1}+\mu_{1}+\theta\right)-\mu_{1} r_{0}\right]}\right. \\
& -\theta\left[\frac{S_{1}}{\mu}+\frac{S_{2}}{q_{2} \lambda_{2}+\beta}\right]+\left[\left(\frac{1}{\mu}+\frac{\alpha}{\mu\left(q_{2} \lambda_{2}+\beta\right)}\right)\right. \\
& +\frac{S_{1}}{\mu}\left(\frac{q \lambda+\mu+\alpha}{\mu}-\frac{\alpha \beta}{\mu\left(q_{2} \lambda_{2}+\beta\right)}\right) \\
& +\frac{S_{2}}{q_{2} \lambda_{2}+\beta}\left[\frac{q_{2} \lambda_{2} \alpha}{\mu\left(q_{2} \lambda_{2}+\beta\right)}+\frac{\alpha}{\mu}\left[\left(\frac{q \lambda+\mu+\alpha}{\mu}\right)\right.\right. \\
& \left.\left.\left.-\left(\frac{\alpha \beta}{\mu\left(q_{2} \lambda_{2}+\beta\right)}\right)\right]\right]\right] \\
& \left.\times\left[\left(\lambda_{1}+\theta\right)-\frac{\lambda_{1} \mu_{1}}{\left[\left(q_{1} \lambda_{1}+\mu_{1}+\theta\right)-\mu_{1} r_{0}\right]}\right]\right]^{-1} \\
& p_{01}=\frac{1}{\mu}\left[\left(\lambda_{1}+\theta\right)-\frac{\lambda_{1} \mu_{1}}{\left[\left(q_{1} \lambda_{1}+\mu_{1}+\theta\right)-\mu_{1} r_{0}\right]}\right] p_{00} \\
& p_{02}=\left[\left(\lambda_{1}+\theta\right)-\frac{\lambda_{1} \mu_{1}}{\left[\left(q_{1} \lambda_{1}+\mu_{1}+\theta\right)-\mu_{1} r_{0}\right]}\right] \\
& \times \frac{\alpha}{\mu\left(q_{2} \lambda_{2}+\beta\right)} p_{00} \\
& p_{10}=\frac{\lambda_{1}}{\left[\left(q_{1} \lambda_{1}+\mu_{1}+\theta\right)-\mu_{1} r_{0}\right]} p_{00} \\
& p_{11}=\frac{1}{\mu}\left[\left[\frac{q \lambda+\mu+\alpha}{\mu}-\frac{\alpha \beta}{\mu\left(q_{2} \lambda_{2}+\beta\right)}\right]\right. \\
& \left.\times\left[\left(\lambda_{1}+\theta\right)-\frac{\lambda_{1} \mu_{1}}{\left[\left(q_{1} \lambda_{1}+\mu_{1}+\theta\right)-\mu_{1} r_{0}\right]}\right]-\theta\right] p_{00} \\
& p_{12}=\left[\left[\frac{\alpha}{\mu}\left(\frac{q \lambda+\mu+\alpha}{\mu}-\frac{\alpha \beta}{\mu\left(q_{2} \lambda_{2}+\beta\right)}\right)\right]\right. \\
& +\frac{q_{2} \lambda_{2} \alpha}{\mu\left(q_{2} \lambda_{2}+\beta\right)}\left[\left(\lambda_{1}+\theta\right)\right. \\
& \left.\left.-\frac{\lambda_{1} \mu_{1}}{\left[\left(q_{1} \lambda_{1}+\mu_{1}+\theta\right)-\mu_{1} r_{0}\right]}\right]-\theta\right] \frac{1}{q_{2} \lambda_{2}+\beta} p_{00}
\end{aligned}
$$

and $p_{i}=p_{1} R^{i-1} ; i \geq 2$

where

$$
\begin{gathered}
S_{0}=\frac{1}{1-r_{0}}+\frac{r_{21} r_{02}+r_{01}\left(1-r_{2}\right)+r_{01} r_{12}+r_{02}\left(1-r_{1}\right)}{\left(1-r_{0}\right)\left[\left(1-r_{1}\right)\left(1-r_{2}\right)-r_{21} r_{12}\right]} \\
S_{1}=\frac{1-r_{2}+r_{12}}{\left[\left(1-r_{1}\right)\left(1-r_{2}\right)-r_{21} r_{12}\right]} \\
S_{2}=\frac{1-r_{1}+r_{21}}{\left[\left(1-r_{1}\right)\left(1-r_{2}\right)-r_{21} r_{12}\right]}
\end{gathered}
$$

Proof. $p_{00}, p_{01}, p_{02}, p_{10}, p_{11}$ and $p_{12}$ follows from the equations (3.1), (3.6) and (3.7).

Remark 3.4. Even though $R$ in Theorem 3.2 has a nice structure which enables us to make use of the properties like $R^{n}=\left[\begin{array}{cc}r_{0}^{n} & r_{01} \sum_{j=0}^{n-1} r_{0}^{j} r_{1}^{n-j-1} \\ 0 & r_{1}^{n}\end{array}\right]$, for $n \geq 1$, due to the form of $r_{0} \& r_{01}$, it may not be easy to cary out the computation required to calculate the $p_{i}$ and the performance measures. Hence, we explore the possibility of algorithmic computation of $R$. The computation of $R$ can be carried out using a number of well-known methods. We use Theorem 1 of [15]. The matrix $R$ is computed by successive substitutions in the recurrence relation:

$$
\begin{aligned}
R(0) & =0 \\
R(n+1) & =-A_{0} A_{1}^{-1}-[R(n)]^{2} A_{2} A_{1}^{-1}, \text { for } n \geq 0
\end{aligned}
$$

and is the limit of the monotonically increasing sequence of matrices $\{R(n), n \geq 0\}$.

\section{Performance Measures}

Using straightforward calculations the following performance measures have been obtained:

(i) Mean queue length $E(L)=p_{0} R(I-R)^{-2} e$

(ii) $E\left(L^{2}\right)=p_{0} R(I+R)(I-R)^{-3} e$

(iii) Variance of $L=\operatorname{var}(L)=p_{0} R\left\{(I+R)-p_{0} R(I-R)^{-1} e\right\}$ $(I-R)^{-3} e$

(iv) Probability that the server is ideal $=p_{0} e$

(v) Mean queue length when the server is an vacation period $=\sum_{i=0}^{\infty} i p_{i 0}$

(vi) Mean queue length when the server is in regular busy period $=\sum_{i=0}^{\infty} i p_{i 1}$

(vii) Probability that the server is in working vacation period $=\operatorname{pr}\{J=0\}=\sum_{i=1}^{\infty} p_{i 0}$

(viii) Probability that the server is in regular busy period $=p r\{J=1\}=\sum_{i=1}^{\infty} p_{i 1}$ 


\section{Particular Model}

In the above model, we assume that $\lambda_{1}=\lambda_{2}=\lambda, q_{1}=$ $q_{2}=q$ and $\mu_{1}=\mu_{2}=\mu$, then we get

$$
\begin{aligned}
& p_{00}=\left[1+\frac{S_{0} \lambda}{\left[(q \lambda+\mu+\theta)-\mu r_{0}\right]}-\theta\left[\frac{S_{1}}{\mu}+\frac{S_{2}}{q \lambda+\beta}\right]\right. \\
& +\left[\left(\frac{1}{\mu}+\frac{\alpha}{\mu(q \lambda+\beta)}\right)+\frac{S_{1}}{\mu}\left(\frac{q \lambda+\mu+\alpha}{\mu}\right.\right. \\
& \left.-\frac{\alpha \beta}{\mu(q \lambda+\beta)}\right)+\frac{S_{2}}{q \lambda+\beta}\left[\frac{q \lambda \alpha}{\mu(q \lambda+\beta)}\right. \\
& \left.\left.+\frac{\alpha}{\mu}\left[\left(\frac{q \lambda+\mu+\alpha}{\mu}\right)-\left(\frac{\alpha \beta}{\mu(q \lambda+\beta)}\right)\right]\right]\right] \\
& \left.\times\left[(\lambda+\theta)-\frac{\lambda \mu}{\left[(q \lambda+\mu+\theta)-\mu r_{0}\right]}\right]\right]^{-1} \\
& p_{01}=\frac{1}{\mu}\left[(\lambda+\theta)-\frac{\lambda \mu}{\left[(q \lambda+\mu+\theta)-\mu r_{0}\right]}\right] p_{00} \\
& p_{02}=\frac{\alpha}{\mu(q \lambda+\beta)}\left[(\lambda+\theta)-\frac{\lambda \mu}{\left[(q \lambda+\mu+\theta)-\mu r_{0}\right]}\right] p_{00} \\
& p_{10}=\frac{\lambda}{\left[(q \lambda+\mu+\theta)-\mu r_{0}\right]} p_{00} \\
& p_{11}=\frac{1}{\mu}\left[\left[\frac{q \lambda+\mu+\alpha}{\mu}-\frac{\alpha \beta}{\mu(q \lambda+\beta)}\right]\right. \\
& \left.\times\left[(\lambda+\theta)-\frac{\lambda \mu}{\left[(q \lambda+\mu+\theta)-\mu r_{0}\right]}\right]-\theta\right] p_{00} \\
& p_{12}=\left[\left[\frac{q \lambda \alpha}{\mu(q \lambda+\beta)}+\frac{\alpha}{\mu}\left(\frac{q \lambda+\mu+\alpha}{\mu}-\frac{\alpha \beta}{\mu(q \lambda+\beta)}\right)\right]\right. \\
& \left.\times\left[(\lambda+\theta)-\frac{\lambda \mu}{\left[(q \lambda+\mu+\theta)-\mu r_{0}\right]}\right]-\theta\right] \\
& \times \frac{1}{q \lambda+\beta} p_{00}
\end{aligned}
$$

and $p_{i}=p_{1} R^{i-1} ; i \geq 2$

\section{Numerical Study}

In this section, some examples are given to show the effect of the parameters $\lambda, \lambda_{1}, \lambda_{2}, \mu, \mu_{1}, q, q_{1}, q_{2}$ and $\theta$ on the performance measures mean queue length, $E\left(L^{2}\right)$, variance of $\mathrm{L}$, probability that the server is idle, mean queue length when the server is an vacation period, mean queue length when the server is regular busy period, probability that the server is in working vacation period and probability that the server is in regular busy period for the model analyzed in this paper. The corresponding results are presented as case(1), case(2) and case(3).
Case(1): If $\lambda=0.5, \lambda_{1}=0.3, \lambda_{2}=0.1, \mu=5, \mu_{1}=2, \theta=$ 2.8, $\alpha=0.4, \beta=0.8, q=0.6, q_{1}=0.4$ and $q_{2}=0.2$ the matrix $R$ is obtained using the equations (3.9) \& (3.10)

$$
R=\left[\begin{array}{ccc}
0.025704 & 0.013719 & 0.006692 \\
0 & 0.06 & 0.029268 \\
0 & 0.004 & 0.026341
\end{array}\right]
$$

and the invariant probability vector is $P=\left(p_{0}, p_{1}, p_{2}, \ldots\right)$ where

$$
\begin{aligned}
& p_{0}=(0.497847,0.275955,0.134612) \\
& p_{1}=(0.031991,0.034170,0.019952)
\end{aligned}
$$

and the remaining vectors $p_{i}^{\prime}$ s are evaluated using the relation

$$
p_{i}=p_{1} R^{i-1}, \text { for } i \geq 2
$$

$p_{2}=(0.000822296715,0.002568892669,0.001739726984)$

$p_{3}=(0.000021136315,0.000172373562,0.000126515311)$

$p_{4}=(0.000000543288,0.000011138443,0.000008519013)$

$p_{5}=(0.000000013965,0.000000709836,0.000000554035)$

$p_{6}=(0.000000000359,0.000000044998,0.000000035463)$

$p_{7}=(0.000000000009,0.000000002847,0.000000002254)$

$p_{8}=(0.000000000001,0.000000000180,0.000000000143)$

$$
\begin{aligned}
p_{9} & =(0,0.000000000011,0.000000000009) \\
p_{10} & =(0,0.000000000001,0.000000000001)
\end{aligned}
$$

For the chosen parameters $p_{10} \rightarrow 0$, and the sum of the steady state probabilities is found to be 0.999999

The performance measures are

(i) Mean queue length $E(L)=0.060371$

(ii) $E\left(L^{2}\right)=0.069080$

(iii) Variance of $\mathrm{L}=\operatorname{var}(L)=0.065435$

(iv) Probability that the server is ideal $=0.908414$

(v) Mean queue length when the server is an vacation period $=0.033701$

(vi) Mean queue length when the server is regular busy period $=0.039873$

(vii) Probability that the server is in working vacation period $=\operatorname{pr}\{J=0\}=0.032835$

(viii) Probability that the server is in regular busy period $=\operatorname{pr}\{J=1\}=0.036923$

Case(2): If $\lambda=0.7, \lambda_{1}=0.5, \lambda_{2}=0.3, \mu=6, \mu_{1}=3, \theta=$ 2.2, $\alpha=0.5, \beta=0.8, q=0.5, q_{1}=0.3$ and $q_{2}=0.1$ the matrix $R$ is obtained using the equations (3.9) \& (3.10)

$$
R=\left[\begin{array}{ccc}
0.028493 & 0.010754 & 0.006478 \\
0 & 0.058333 & 0.035140 \\
0 & 0.005 & 0.039157
\end{array}\right]
$$

and the invariant probability vector is 
where

$$
\begin{aligned}
& p_{0}=(0.538051,0.216572,0.130465) \\
& p_{1}=(0.051102,0.032573,0.024338)
\end{aligned}
$$

and the remaining vectors $p_{i}^{\prime}$ s are evaluated using the relation

$$
p_{i}=p_{1} R^{i-1}, \text { for } i \geq 2
$$

$p_{2}=(0.001456049387,0.002571321791,0.002428656910)$

$p_{3}=(0.000041487216,0.000177794544,0.000194887456)$

$p_{4}=(0.000001182095,0.000011791880,0.000014147662)$

$p_{5}=(0.000000033681,0.000000771306,0.000000976004)$

$p_{6}=(0.000000000960,0.000000050235,0.000000065539)$

$p_{7}=(0.000000000027,0.000000003268,0.000000004338)$

$p_{8}=(0.000000000001,0.000000000213,0.000000000285)$

$$
\begin{aligned}
p_{9} & =(0,0.000000000014,0.000000000019) \\
p_{10} & =(0,0.000000000001,0.000000000001)
\end{aligned}
$$

For the chosen parameters $p_{10} \rightarrow 0$, and the sum of the steady state probabilities is found to be 0.999999

The performance measures are

(i) Mean queue length $E(L)=0.057692$

(ii) $E\left(L^{2}\right)=0.065529$

(iii) Variance of $\mathrm{L}=\operatorname{var}(L)=0.062201$

(iv) Probability that the server is ideal $=0.885088$

$(v)$ Mean queue length when the server is an vacation period $=0.054143$

(vi) Mean queue length when the server is regular busy period $=0.038301$

(vii) Probability that the server is in working vacation period $=\operatorname{pr}\{J=0\}=0.052601$

(viii) Probability that the server is in regular busy period $=\operatorname{pr}\{J=1\}=0.035335$

Case(3): If $\lambda_{1}=\lambda_{2}=\lambda=0.3, \mu_{1}=\mu=2, \theta=4.2, \alpha=$ $0.3, \beta=0.6$, and $q_{2}=q_{1}=q=0.5$ the matrix $R$ is obtained using the equations (3.9) \& (3.10)

$$
R=\left[\begin{array}{ccc}
0.0238 & 0.0512 & 0.02048 \\
0 & 0.075 & 0.03 \\
0 & 0.075 & 0.23
\end{array}\right]
$$

and the invariant probability vector is where

$$
\begin{aligned}
& p_{0}=(0.202085,0.445072,0.178029) \\
& p_{1}=(0.009619,0.067426,0.062576)
\end{aligned}
$$

and the remaining vectors $p_{i}^{\prime}$ s are evaluated using the relation

$$
p_{i}=p_{1} R^{i-1}, \text { for } i \geq 2
$$

$$
\begin{array}{r}
p_{2}=(0.000228932215,0.010242643766,0.016612257808) \\
p_{3}=(0.000005448587,0.002025838941,0.004132787231) \\
p_{4}=(0.000000129676,0.000462175958,0.001011427841) \\
p_{5}=(0.000000003086,0.000110526926,0.000246496347) \\
p_{6}=(0.000000000073,0.000026776905,0.000060010032) \\
p_{7}=(0.00000000002,0.000006509024,0.000014605616) \\
p_{8}=(0,0.000001583598,0.000003554563) \\
p_{9}=(0,0.000000385362,0.000000865057) \\
p_{10}=(0,0.000000093781,0.000000210524)
\end{array}
$$

For the chosen parameters $p_{10} \rightarrow 0$, and the sum of the steady state probabilities is found to be 0.999999

The performance measures are

(i) Mean queue length $E(L)=0.193337$

(ii) $E\left(L^{2}\right)=0.301677$

(iii) Variance of $\mathrm{L}=\operatorname{var}(L)=0.244298$

(iv) Probability that the server is ideal $=0.825186$

(v) Mean queue length when the server is an vacation period $=0.010094$

(vi) Mean queue length when the server is regular busy period $=0.096633$

(vii) Probability that the server is in working vacation period $=\operatorname{pr}\{J=0\}=0.009854$

(viii) Probability that the server is in regular busy period $=\operatorname{pr}\{J=1\}=0.080302$

\section{Conclusion}

A single server Markovian working vacation queue with customer balking and with Breakdown has been analyzed in this article. In addition the arrival rates depends on the server state. The model can be generalized by taking arrival time/service time/breakdown period/repair period follows a general distribution.

\section{Acknowledgment}

The work of the second author was supported by University Grants Commission, New Delhi through the BSR (Fellowship) Grant No. F.25-1/2014-15(BSR)/7-254/2009.

\section{References}

[1] Abou, El-Ata., M.O. and Hariri, A.M.A., The $M / M / c / N$ queue with balking and reneging, Computers and Operation Research, 19(1992), 713-716.

[2] Ancker, Jr., C.J. and Gafarian, A.V., Some queueing problems with balking and reneging;I, Operations Research, 11(1963a), 88-100. 
[3] Ancker, Jr., C.J. and Gafarian, A.V., Some queueing problems with balking and reneging;II, Operations Research, 11(1963b), 928-937.

[4] Avi-Itzhak, B. and Naor, P., Some queueing problems with the service station subject to breakdowns, Operation Research 11(1963), 303-320.

[5] Barrer, D.Y., Queueing with impatient customers and Indifferent clerks, Oper. Res., 5(1957), 644-649.

[6] Doshi, B.T., Queueing systems with vacations-a survey, Queueing systems 1(1986), 29-66.

[7] Federgruen, A. and Green, L., Queueing system with service interruptions, Operation Research 34(1986), 752768

[8] Fond, S. and Ross, S., A heterogeneous arrival and service queueing loss model, Tech-Report ORC 77-12, Operations Research Center, University of California, Berkeley, CA, 1977.

[9] Gaver, D.P., A waiting line with interrupted service including priorities, Franian, Jr. R. Stat. Soc., ser-B 24(1962), 73-90.

[10] Haight, F.A., Queueing with Balking, Biometrika 46(1957), 360-369.

[11] Jain, M. and Jain, A., Working vacation queueing model multiple type of server breakdown, Appl. math. modelling, 34(1)(2010), 1-13, 2010.

[12] Jayaraman, D., R. Nadarajan and M.R. Sitrarasu, A general bulk service queue with arrival rate dependent on server breakdowns, Appl. math. modelling, 18(1994), $156-160$.

[13] Ke, J.C, Wu, C.H, and Zhang, Z.G., Recent development in vacation queueing models: A short surrey, Int. Jr. of Oper. Res., 7(4)(2010), 3-8.

[14] Kim, J.D., Choi, D.W., and Chae, K.C., Analysis of queue-length distribution of the $M / G / 1$ with working vacation, $(M / G / 1 / W V)$, In Proceeding of the International Conference on Statistics and related, Honolulu, Hawaii, USA, 2003.

[15] Latouche, G and Neuts, M.F., Efficient algorithmic solutions to exponential tandem queues with blocking, SIAM J. Algebraic Discrete Math., 1(1980), 93-106.

[16] Li, J.H, Tian, N., Zhang, Z.G., and Luh, H.P., Analysis of the $M / G / 1$ queue with exponenetially working vacationa matrix analytic approach, Queueing systems, 61(2009), $139-166$.

[17] Lin, Ch.H., and Ke, J.Ch., Multi-server system with single working vacation, Applied Mathematical Modelling, 33(2009), 2967-2977.

[18] Liu, W., Xu, X. and Tian, N., Stochastic decompositions in the $M / M / 1$ queue with working vacation, Oper. Res. Lett., 35(2007), 595-600.

[19] Lucantoni, D.M., A $G I / M / C$ queue with a different service rate for customers who need not wait an algorithmic solution, Technical Rep. Univ. of Delware, USA, (1979).

[20] Neuts, M.F., Markov chains with applications in queueing theory which have a matrix-geometric invariant probabil- ity vector, Adv. Appl. Probab., 10(1978), 185-212.

[21] Neuts, M.F, Matrix-Geometric solution in stochastic models, Vol 2 of John Hopkins series in the Mathematical Sciences, Johns Hopkins University press, Baltimore, md, USA, 1981.

[22] Servi, L.D., and Finn, S.G., $M / M / 1$ queues with working vacations $(M / M / 1 / W V)$, Perform. Eval. 50(2002), 41$52,2002$.

[23] Shanthikumar, J.G., Analysis of a single server queue with time and operational dependent server failures, $A d v$. in mgnt. studies, 1(1982), 339-359.

[24] Sherif, I.Ammar., Mohmoud, M.Helan., and Faizah, T.Alamri., The busy period of an $M / M / 1$ queue with balking and reneging, Applied mathematical modelling. 37(2013), 9223-9229.

[25] Shogan, A.W., A single server queue with arrival rate dependent on server breakdowns, Naval Res. Log. Quart. 26(1979), 487-497.

[26] Takagi, H., Queueing Analysis-A Foundation of Performance Evaluation vacation and Priority Systems, Vol. 1, North-Holland, New York, 1991.

[27] Thiruvengadam, K., Queueing with breakdowns, Operation Research 11(1963), 303-320.

[28] Tian. N and Yue. D, Quasi-birth and death process and the matrix geometric solution, Beijing: Science Press, 2002.

[29] Vam Dijk, N., Simple bounds for queueing systems with breakdowns, Performance Evaluation 8(2)(1988), 117128.

[30] Wang, K.H., and Chang, Y.C., Cost analysis of a finite $M / M / R$ queueing system with balking, reneging and server breakdown, Mathematical methods of Operations Research. 56(2)(2002), 169-180.

[31] White, H. and Christie, L., Queueing with pre-emptive priorities or with breakdown, Operation Research 6(1958), 79-95.

[32] Wu, D.A., and Takagi, H., $M / G / 1$ queue with multiple working vacations, Perform. Eval. 63(2006), 654-681.

[33] Xu, X., Zhang, Z. and Tian, N. Analysis for the $M^{X} / M / 1$ working vacation queue, Int. Jr. of Infor. \& Manag. Sci., 20(2009), 3, 379-394.

[34] Yechiali, V and Naor. P., Queueing problems with heterogeneous arrivals and service, Oprs. Res, 19(1971), $722-734$. 